\title{
Anisotropic behaviours of massless Dirac fermions in graphene under periodic potentials
}

\author{
CHEOL-HWAN PARK ${ }^{1,2}$, LI YANG ${ }^{1,2}$, YOUNG-WOO SON ${ }^{3}$, MARVIN L. COHEN ${ }^{1,2}$ AND STEVEN G. LOUIE1,2* \\ ${ }^{1}$ Department of Physics, University of California at Berkeley, Berkeley, California 94720, USA \\ ${ }^{2}$ Materials Sciences Division, Lawrence Berkeley National Laboratory, Berkeley, California 94720, USA \\ ${ }^{3}$ Department of Physics, Konkuk University, Seoul 143-701, Korea \\ *e-mail: sglouie@berkeley.edu
}

Graphene's conical valence and conduction bands give rise to charge carriers that have neutrino-like linear energy dispersion and exhibit chiral behaviour near the Dirac points where these bands meet ${ }^{1-6}$. Such characteristics offer exciting opportunities for the occurrence of new phenomena and the development of high performance electronic devices. Making high quality devices from graphene, which typically involves etching it into nanoscale structures ${ }^{7-10}$, however, has proven challenging. Here we show that a periodic potential applied by suitably patterned modifications or contacts on graphene's surface leads to further unexpected and potentially useful charge carrier behaviour. Owing to their chiral nature, the propagation of charge carriers through such a graphene superlattice is highly anisotropic, and in extreme cases results in group velocities that are reduced to zero in one direction but are unchanged in another. Moreover, we show that the density and type of carrier species (electron, hole or open orbit) in a graphene superlattice are extremely sensitive to the potential applied, and they may further be tuned by varying the Fermi level. As well as addressing fundamental questions about how the chiral massless Dirac fermions of graphene propagate in a periodic potential, our results suggest the possibility of building graphene electronic circuits from appropriately engineered periodic surface patterns, without the need for cutting or etching.

Since the pioneering work by Esaki and $\mathrm{Tsu}^{11}$, superlattices have been studied extensively and have had a huge impact on semiconductor physics ${ }^{12,13}$. Superlattices demonstrate interesting phenomena such as negative differential conductivity, Bloch oscillations, gap openings at the mini-Brillouin-zone boundary formed by the additional periodic potential and so on ${ }^{12,13}$. Conventional semiconducting superlattices are mainly produced by molecular-beam epitaxy and metallo-organic chemical vapourphase deposition, whereas metallic superlattices are made by sputtering procedures ${ }^{12,13}$. We expect that, by modulating the potential seen by the electrons, graphene superlattices may be fabricated by adsorbing adatoms on graphene surface through similar techniques, by positioning and aligning impurities with scanning tunnelling microscopy ${ }^{14-16}$ or by applying a local topgate voltage to graphene ${ }^{17-19}$. Epitaxial growth of graphene $e^{5}$ on top of a prepatterned substrate is also a possible route to a graphene superlattice. Recently, a periodic pattern in the scanning tunnelling microscope image has been demonstrated on a graphene monolayer on top of a metallic substrate $e^{20-22}$ as well. On the theoretical side, it has been shown that a triangular array of capped carbon nanotubes embedded vertically in graphene is semiconducting and may become ferromagnetic on doping ${ }^{23}$, and corrugation of graphene is expected to induce inhomogeneous charge densities and localized states ${ }^{24}$.

The low-energy charge carriers in pristine graphene are described by a massless Dirac equation and have a linear energy dispersion which is isotropic near the Dirac points $\mathrm{K}$ and $\mathrm{K}^{\prime}$ in the Brillouin zone $e^{1,3,4,25-27}$ (Fig. 1a). It is shown experimentally that the carriers have a group velocity of $v_{0} \approx 10^{6} \mathrm{~m} \mathrm{~s}^{-1}$, which plays the role of an effective speed of light in $(2+1)$-dimensional quantum electrodynamics ${ }^{3,4}$. Within the effective-hamiltonian formalism, the wavefunction of the quasiparticles in graphene has two components corresponding to the amplitude on the two different trigonal sublattices of graphene, and can be represented by a twocomponent spinor ${ }^{25-27}$. This spinor structure of the wavefunction is called a pseudospin (because it is not related to a real spin) or chirality $^{25-29}$, which is of central importance to the novel physical properties of graphene superlattices discussed below.

Let us now consider the situation where an additional periodic potential is applied to graphene. If the spatial period of the superlattice potential is much larger than the nearest-neighbour carbon-carbon distance in graphene $(\sim 1.42 \AA)$, the scattering of a state close to one Dirac point to another one does not occur ${ }^{25,26,28}$. Therefore, even though there are two non-equivalent Dirac cones for the energy dispersion surface of graphene, focusing on a single cone is sufficient. This condition also implies that, in the graphene superlattices discussed here, there is no gap opening at the Dirac point ${ }^{25,26,28}$.

To investigate the physics of charge carriers in graphene superlattices, we have calculated the energy dispersions, the group velocities and the energy-gap openings at the minizone boundary (MB) within the effective-hamiltonian formalism ${ }^{1}$. Effects of the external periodic potential are incorporated into our calculation through the scattering matrix elements between pseudospin states, or chiral eigenstates, of the electrons in graphene (see the Methods section). We have also carried out a tight-binding formulation and obtained results identical to those discussed below.

First, for a one-dimensional (1D) graphene superlattice (Fig. 1b), we find that the group velocity for states with wavevector $\mathbf{k}$ ( $\mathbf{k}$ is the wavevector of the Bloch state defined with respect to 
a

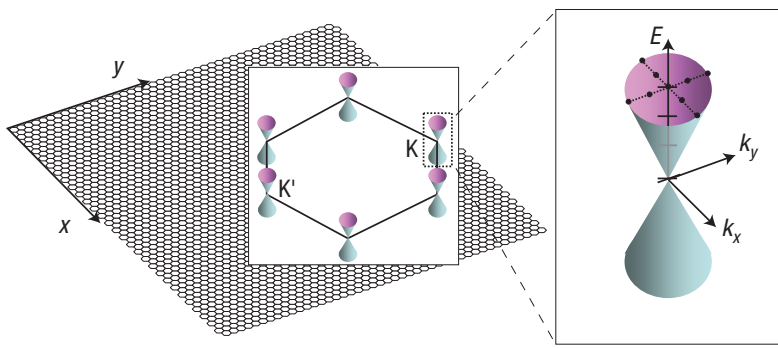

b
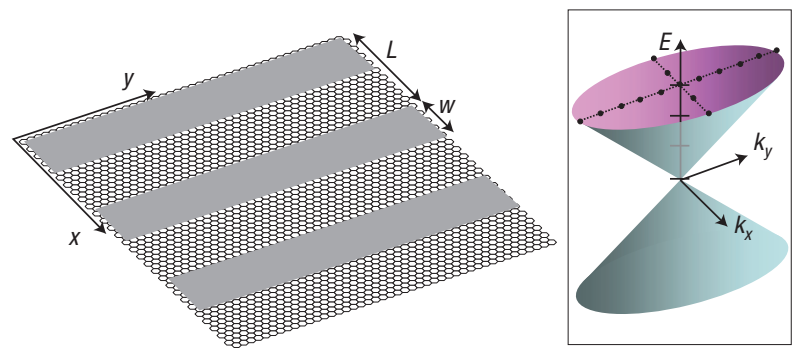

G

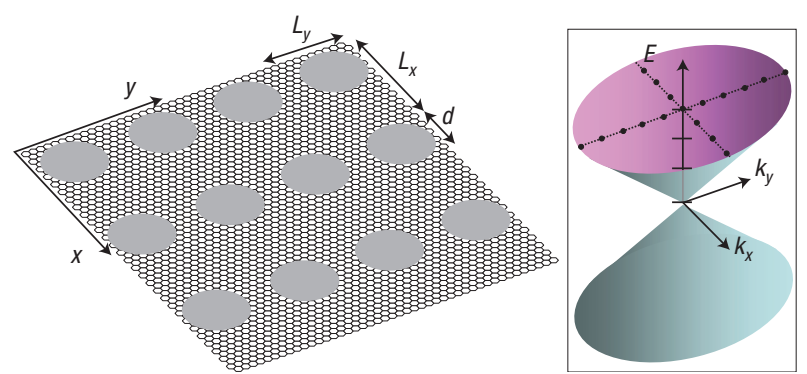

Figure 1 Graphene superlattices and anisotropic Dirac cones. a, Schematic diagram of graphene. Inset: The Brillouin zone of graphene and Dirac cones centred at Dirac points, among which two ( $K$ and $K^{\prime}$ ) are non-equivalent (left), and the linear and isotropic energy dispersion near one of the Dirac points of charge carriers in graphene (right). $\mathbf{b}$, One-dimensional (1D) graphene superlattice formed by a Kronig-Penney type of potential periodic along the $\hat{x}$ direction with spatial period $L$ and barrier width $w$. The potential is $U_{10}$ in the grey regions and zero outside. Inset: Energy dispersion of charge carriers in the $1 \mathrm{D}$ graphene superlattice. The energy dispersion along any line in two-dimensional (2D) wavevector space from the Dirac point is linear but with a different group velocity. For a particle moving parallel to the periodic direction the group velocity $\left(\boldsymbol{v}_{\|}\right)$is not renormalized at all, whereas that for a particle moving perpendicular to the periodic direction $\left(v_{\perp}\right)$ is reduced most. c, $2 \mathrm{D}$ graphene superlattice with a muffin-tin type of potential periodic along both $\hat{x}$ and $\hat{y}$ directions with spatial periods $L_{x}$ and $L_{y}$, respectively. The potential is $U_{20}$ inside the grey disks with diameter $d$ and zero outside. Inset: Energy dispersions of charge carriers in the $2 \mathrm{D}$ graphene superlattice.

the Dirac point) is anisotropically renormalized, that is, it is a strong function of the direction of $\mathbf{k}$. For pristine graphene, the group velocity of states near the Dirac point is parallel to $\mathbf{k}$ and of constant magnitude $\left(v_{0}\right)$. For example, in a 1D superlattice of a Kronig-Penney type of periodic potential with potential barrier height $\left(U_{1 \mathrm{D}}\right)$ of $0.5 \mathrm{eV}$ and spatial period $(L)$ and barrier width $(w)$ of $10 \mathrm{~nm}$ and $5 \mathrm{~nm}$, respectively, the group velocity of the charge carriers when $\mathbf{k}$ is along a certain direction is renormalized to be less than $40 \%$ of its original value $v_{0}$, but it is the same as $v_{0}$ along some other direction. (Figure 2a: the plotted quantity a

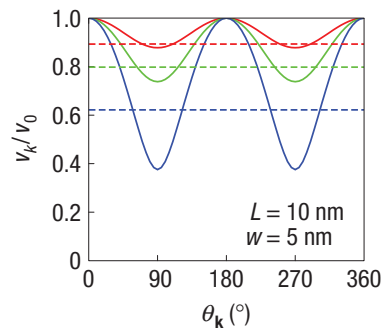

c

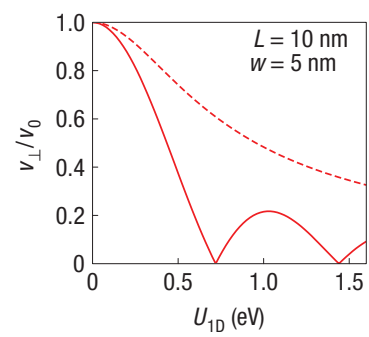

b

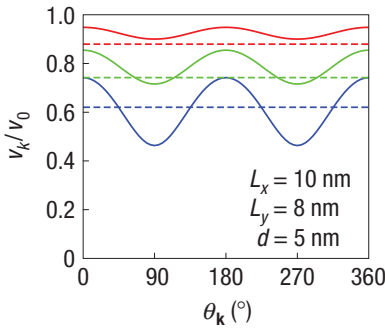

d

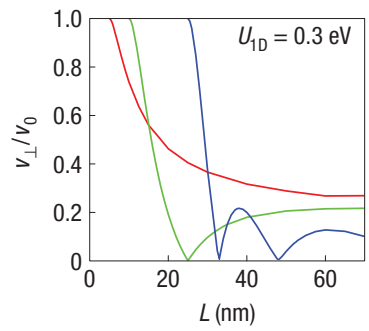

Figure 2 Anisotropic velocity renormalization in graphene superlattices. a, The component of the group velocity parallel to the $\mathbf{k}$ vector $\left(v_{\hat{k}} \equiv \mathbf{v}(\mathbf{k}) \cdot \hat{k}\right.$ with $\mathbf{k}$ measured from the Dirac point) of charge carriers in a $1 \mathrm{D}$ graphene superlattice in units of the Fermi velocity in graphene $\left(v_{0}\right)$ versus the angle $\left(\theta_{\mathbf{k}}\right)$ of the $\mathbf{k}$-vector from the periodic potential direction $\hat{x}$ (solid lines) and that in a superlattice made from a fictitious system of non-chiral fermions with properties otherwise identical to those in graphene (dashed lines). Red, green and blue lines correspond to $U_{10}$ being $0.2 \mathrm{eV}, 0.3 \mathrm{eV}$ and $0.5 \mathrm{eV}$, respectively. b. Similar quantities as in a for a rectangular $2 \mathrm{D}$ graphene superlattice. Red, green and blue lines correspond to $U_{20}$ being $0.3 \mathrm{eV}$, $0.5 \mathrm{eV}$ and $0.7 \mathrm{eV}$, respectively. c, The group velocity of charge carriers in a 1D graphene superlattice with $\mathbf{k}$ perpendicular to the periodic direction, $v_{\perp}$, in units of $v_{0}$, versus $U_{10}$ (solid line), and that in a superlattice made from a fictitious system of non-chiral fermions with properties otherwise identical to those in graphene (dashed line). d, $v_{\perp}$ versus the potential spatial period $(L)$ of charge carriers in a $1 D$ graphene superlattice. Red, green and blue lines correspond to a fixed potential barrier height but with width (w) being $5 \mathrm{~nm}, 10 \mathrm{~nm}$ and $25 \mathrm{~nm}$, respectively.

$v_{\hat{k}} \equiv \mathbf{v}(\mathbf{k}) \cdot \hat{k}$ is the component of the group velocity parallel to the wavevector $\mathbf{k}$ in units of $v_{0}$. We note that this quantity, which depends only on the direction of $\mathbf{k}$ (see Supplementary Information, Discussion S1), is exactly equal to the absolute value of the group velocity $v_{\mathrm{g}}$ when $\mathbf{k}$ is at $0^{\circ}, 90^{\circ}, 180^{\circ}$ or $270^{\circ}$ from the periodic direction of the applied potential and, when the applied potential is weak, is only slightly different from $v_{\mathrm{g}}$ at other angles (see Supplementary Information, Discussion S2).) Thus, the group velocity of charge carriers can be tailored highly anisotropically in graphene superlattices. More interestingly, the group velocity when $\mathbf{k}$ is along the direction perpendicular to the periodic direction of the potential $\left(v_{\perp}\right)$ is reduced the most, whereas when $\mathbf{k}$ is in the parallel direction it is not renormalized at all (Fig. 1b). This result is counter-intuitive, because the velocity is strongly reduced when the charge carrier is moving parallel to the hurdles, but is not modulated when it is crossing them.

To understand the physics behind this phenomenon, we have carried out the same calculation for a fictitious system with carriers that have no chirality but are otherwise identical to those in graphene, including the linear energy dispersion. The group velocity in this system is reduced isotropically and the renormalized group velocity is close to $v_{\perp}$, that is, the maximally renormalized one, in 1D graphene superlattices (Fig. 2a). Thus, it is clear that the absence of velocity renormalization in the direction parallel to the periodicity of the external potential originates from the chiral 
nature of the electronic states of graphene. This behaviour can be demonstrated more clearly by second-order perturbation theory in the case of the $1 \mathrm{D}$ periodic potential with weak amplitudes (see Supplementary Information, Discussion S1). We note that the chirality discussed here is also important in the tunnelling phenomenon in graphene through a single barrier ${ }^{29}$ or a finite number of barriers ${ }^{30}$.

In the case of $2 \mathrm{D}$ graphene superlattices, the group velocity is renormalized for $\mathbf{k}$ states along every direction, but anisotropically (Fig. 1c). As the amplitude of the potential increases, the overall group velocity is reduced and the ratio of the maximum group velocity to the minimum one is enhanced (Fig. 2b). Here, again, the anisotropy disappears if the chiral nature of the states in graphene is arbitrarily removed. As was demonstrated for the $1 \mathrm{D}$ superlattice, the sinusoidal dependence on the angle of propagation as well as the overall shift in the case of a $2 \mathrm{D}$ graphene superlattice of the component of the renormalized group velocity parallel to $\mathbf{k}$ in the weak-potential limit is well explained and reproduced by second-order perturbation theory (see Supplementary Information, Discussion S1).

Remarkably, the anisotropy in energy dispersions of the 1D superlattices can be tuned by changing the applied potential in such a way that $v_{\perp}$ is reduced completely to zero (Fig. 2c). Hence, we can achieve extremely low mobility in one direction and normal conduction in another one simultaneously. This enables us to control the flow of electrons dramatically. It also provides a novel non-destructive pathway to make graphene nanoribbons ${ }^{7-10}$, which has been actively pursued by way of cutting graphene sheets ${ }^{9,10}$. The chiral nature of the states in graphene also plays a decisive role here. In the model without chirality as discussed before the (isotropic) group velocity of charge carriers is reduced monotonically and never reaches zero within a conceivable range of the potential amplitude (Fig. 2c). We can also achieve vanishing group velocity in one direction by changing the length parameters of the superlattice (Fig. 2d).

Graphene superlattices show peculiar behaviour of gap openings at the MB formed by the external periodic potential (Fig. 3). In conventional layer-structured 1D superlattices, gap opening at the $\mathrm{MB}$ is considered to be nearly constant, independent of $\mathbf{k}$. 1D graphene superlattices, however, are different in that the gap $(\Delta E)$ vanishes when $\mathbf{k}$ is along the direction of the periodic potential, that is, at the centre of the MB (Figs 3a, 4a). Moreover, the size of the gap depends strongly on where it is on the MB (Fig. 3b). These strong anisotropies in the gap opening do not happen in superlattices made from a system with linear energy dispersions but no chirality (Fig. 3b). Hence, again, the chiral nature of charge carriers in graphene is key in generating these anisotropies in the gap opening, as it does in the velocity renormalization. In particular, the gap closure at the centre of the $\mathrm{MB}$ is directly related to the absence of back-scattering of charge carriers from a scattering potential when the size of the scatterer is several times larger than the inter-carbon distance $e^{25,26,28}$. In 1D graphene superlattices, the important length-scale is $L$, which is much larger than the intercarbon distance, and hence the gap does not open at the centre of the MB.

The largest gap at the $\mathrm{MB}$ in a graphene superlattice is proportional to the amplitude of the applied potential if the potential is weak (that is, small compared to the band width) and its size thus can be made to be a few tenths of an electron volt with appropriate perturbation and far above room temperature (Fig. 3b, Supplementary Information, Discussion S4). We have also investigated the gap opening in $1 \mathrm{D}$ graphene superlattices with different values of length parameters $(L$ and $w)$. We find that, by changing these parameters, the anisotropy in the gap at the MB can also be controlled (see Supplementary Information, Discussion S4).
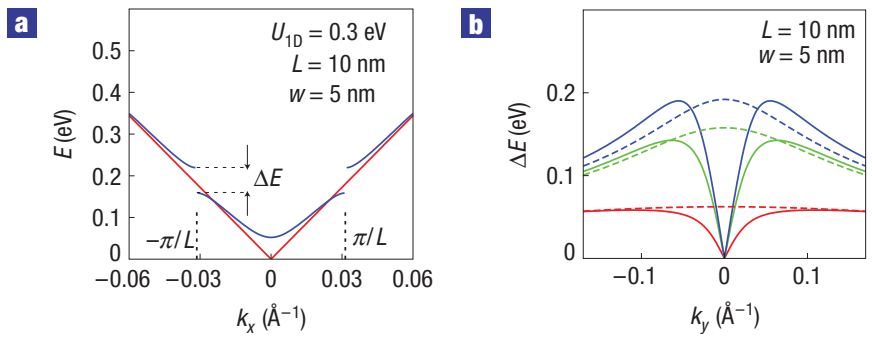

Figure 3 Energy gap at the superlattice Brillouin zone or MB of a 1D graphene superlattice. a, Energy of charge carriers in a 1D graphene superlattice versus the component of the wavevector $\mathbf{k}$ parallel to the periodic potential direction $\left(k_{x}\right)$ at a fixed $k_{y}$. Dashed vertical lines indicate minizone boundaries $\left(k_{x}= \pm \pi / L\right) . \Delta E$ is the energy gap at the minizone boundary for a given $k_{y}$. Red and blue lines correspond to $k_{y}$ being zero and $0.012 \AA^{-1}$, respectively. b, $\Delta E$ versus $k_{y}$ for charge carriers in a $1 \mathrm{D}$ graphene superlattice (solid lines) and that in a superlattice made from a fictitious system with states without chirality but otherwise identical to graphene (dashed lines). Red, green and blue lines correspond to $U_{10}$ being $0.1 \mathrm{eV}$, $0.3 \mathrm{eV}$ and $0.5 \mathrm{eV}$, respectively.

Owing to the velocity renormalization near the Dirac point and the strong anisotropy in energy dispersion close to the MB, the type and the density of states (DOS) of charge carriers vary drastically from those in graphene as we vary the Fermi energy (Fig. 4). As the Fermi level changes, the topology of the Fermi surface also shows a dramatic variation (see Supplementary Information, Discussion S5). For example, as the Fermi level increases from the energy at the Dirac point, the charge carriers of a 1D graphene superlattice fill electron orbits and show a linear increase in the DOS with slope larger than that of graphene, but above a certain value the DOS of electron orbits vanishes and charge carriers suddenly fill open orbits and hole pockets. When the Fermi level increases further, charge carriers are in purely open orbits and then the DOS of electron orbits starts to reappear and increases again (Fig. 4b). We expect that the Fermi level in a graphene superlattice can be tuned as in graphene by applying a gate voltage $e^{3,4,18,19}$. Hence, by exploiting the various characteristics of charge carriers and the Fermi-surface topology, we can manipulate a variety of physical properties dominated by the Fermi surface, such as heat capacity, conductance or magnetoresistance, significantly.

The anisotropic gap opening at the $\mathrm{MB}$ and the dramatic variation of the characters of charge carriers with the Fermi energy are also common in 2D graphene superlattices. The gap at the centres of the zone boundaries closes as in $1 D$ graphene superlattices (Fig. 4c). However, the gap at the corners of the 2D MB also disappears. This behaviour, which occurs in rectangular 2D graphene superlattices in general, again has its origin in the chiral nature of charge carriers in graphene (see Supplementary Information, Discussion S3). In a square 2D graphene superlattice, charge carriers are electrons, holes or a mixture of the two depending on the Fermi level (Fig. 4d). For general rectangular 2D graphene superlattices, charge carriers can also be in open orbits.

Here we have presented several novel physical properties of graphene superlattices with Kronig-Penney-type 1D and muffintin-type $2 \mathrm{D}$ potentials. Through additional calculations, we have confirmed that all the salient features of our findings are the same in sinusoidal or gaussian types of graphene superlattices in general as well. The novel properties discovered in the present study thus should be very robust and relevant to experimentally fabricated graphene superlattices. 
a

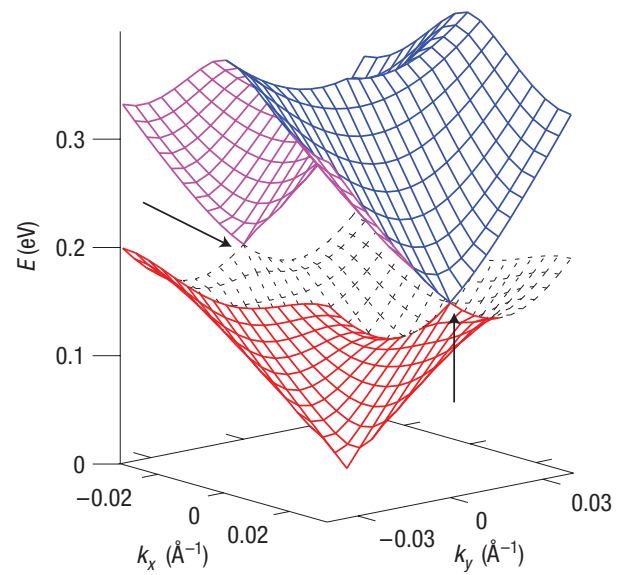

G

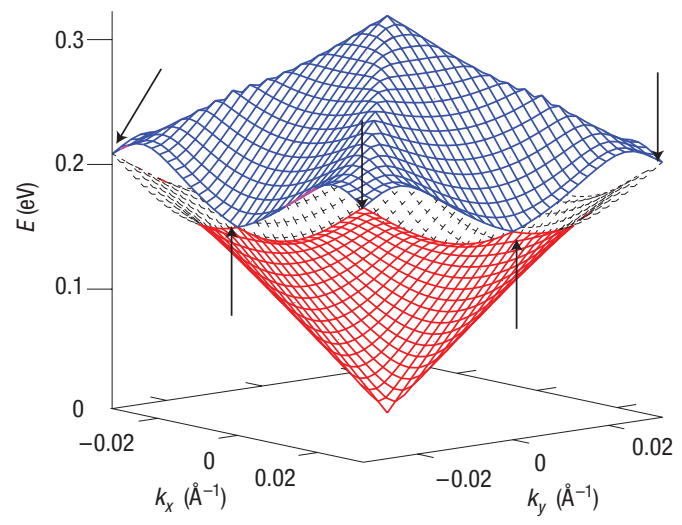

b

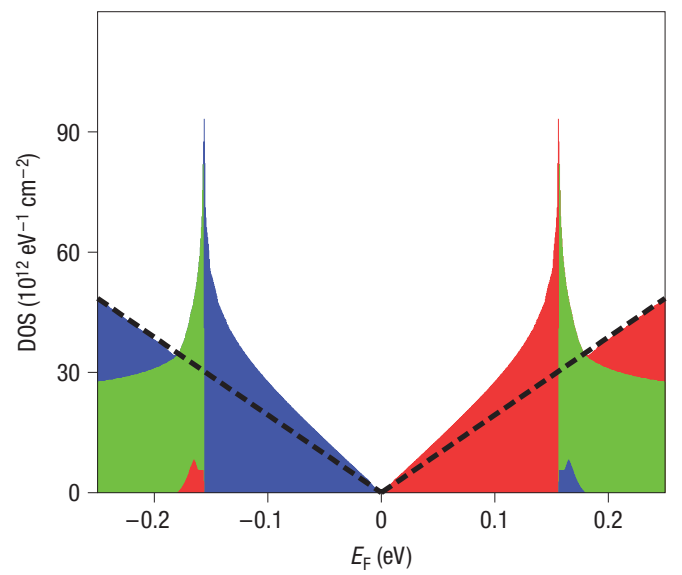

d

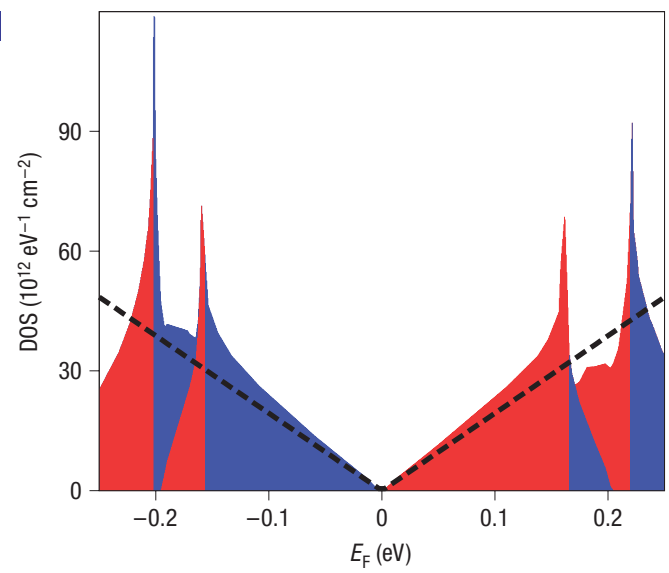

Figure 4 Energy dispersions and DOSs of charge carriers in graphene superlattices. a, Energy of charge carriers in a $1 \mathrm{D}$ graphene superlattice with $U_{10}=0.3 \mathrm{eV}$, $L=10 \mathrm{~nm}$ and $w=5 \mathrm{~nm}$ in the first (red and black) and the second (blue and pink) band above the vertex of the Dirac cone versus 2D wavevector $\mathbf{k}$ away from the Dirac point. MBs are at $k_{x}= \pm 0.031 \AA^{-1}$. Arrows indicate points on the MB where the gap closes. $\mathbf{b}$, DOS of charge carriers in electron orbits (red), open orbits (green) and hole orbits (blue) in the $1 \mathrm{D}$ graphene superlattice characterized in a versus the Fermi energy $\left(E_{F}\right)$. The origin of the energy scale is set at the energy of the Dirac point. The DOS of each species is the height of the corresponding coloured region. The dashed black line shows the DOS of pristine graphene for comparison. c, Similar quantities as in a for a 2D graphene superlattice with $U_{2 D}=0.3 \mathrm{eV}, L_{x}=L_{y}=10 \mathrm{~nm}$ and $d=5 \mathrm{~nm}$. $\mathbf{d}$, Similar quantities as in $\mathbf{b}$ for the $2 \mathrm{D}$ graphene superlattice specified in c.

\section{METHODS}

\section{EFFECTIVE HAMILTONIAN FORMALISM}

There are two carbon atoms per unit cell in graphene, forming two different sublattices, and hence the eigenstate of charge carriers in graphene can be represented by a two-component basis vector. In this work, we are considering eigenstates near the $\mathbf{K}$ point in the Brillouin zone of graphene only as discussed in the paper. The effective hamiltonian for low-energy quasiparticles of graphene in this basis is given by

$$
H_{0}(\mathbf{k})=\hbar v_{0}\left(\begin{array}{cc}
0 & -i k_{x}-k_{y} \\
i k_{x}-k_{y} & 0
\end{array}\right),
$$

where $v_{0}$ is the Fermi velocity and $\mathbf{k}$ the small wavevector of the quasiparticle from the $\mathbf{K}$ point. The energy spectrum of this hamiltonian is $E=s \hbar v_{0} k$, where $s$ is +1 or -1 for an eigenstate above or below the Dirac-point energy, which is defined to be the energy zero, respectively. Eigenstates of this hamiltonian are given by

$$
\langle\mathbf{r} \mid s, \mathbf{k}\rangle=\frac{1}{\sqrt{2}} \mathrm{e}^{i(\mathbf{K}+\mathbf{k}) \cdot \mathbf{r}}\left(\begin{array}{c}
1 \\
i s \mathrm{e}^{i \theta_{\mathbf{k}}}
\end{array}\right),
$$

where $\theta_{\mathbf{k}}$ is the angle of vector $\mathbf{k}$ with respect to the $\hat{k}_{x}$ direction. Now, when an additional periodic potential $U(\mathbf{r})$ is applied to graphene, the scattering amplitude between states is given by

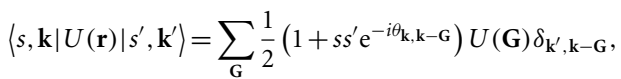

where $\mathbf{G}$ and $U(\mathbf{G})$ are the reciprocal lattice vector and the corresponding Fourier component of the external periodic potential, respectively, and $\theta_{\mathbf{k}, \mathbf{k}-\mathbf{G}}$ the angle from $\mathbf{k}-\mathbf{G}$ to $\mathbf{k}$. The energy dispersions and eigenstates of the quasiparticles in a graphene superlattice are obtained non-perturbatively within the single-particle picture by solving the following set of linear equations:

$$
\left(E-\varepsilon_{s, k}\right) c(s, \mathbf{k})=\sum_{s^{\prime}, \mathbf{G}} \frac{1}{2}\left(1+s s^{\prime} \mathrm{e}^{\left.-i \theta_{\mathbf{k}, \mathbf{k}-\mathbf{G}}\right)} U(\mathbf{G}) c\left(s^{\prime}, \mathbf{k}-\mathbf{G}\right),\right.
$$

where $E$ is the superlattice energy eigenvalue and $\varepsilon_{s, \mathbf{k}}=s \hbar v_{0} k$ the energy of the quasiparticles before applying the periodic potential. $c(s, \mathbf{k})$ and $c\left(s^{\prime}, \mathbf{k}-\mathbf{G}\right)$ are the amplitudes of mixing among different unperturbed quasiparticle states.

Received 3 October 2007; accepted 28 January 2008; published 24 February 2008.

\section{References}

1. Wallace, P. R. The band theory of graphite. Phys. Rev. 71, 622-634 (1947).

2. Novoselov, K. S. et al. Two-dimensional atomic crystals. Proc. Natl Acad. Sci. USA. 102 10451-10453 (2005)

3. Novoselov, K. S. et al. Two-dimensional gas of massless Dirac fermions in graphene. Nature $\mathbf{4 3 8}$ $197-200(2005)$ 
4. Zhang, Y., Tan, J. W., Stormer, H. L. \& Kim, P. Experimental observation of the quantum Hall effect and Berry's phase in graphene. Nature 438, 201-204 (2005).

5. Berger, C. et al. Electronic confinement and coherence in patterned epitaxial graphene. Science 312 $1191-1196$ (2006).

6. Geim, A. K. \& Novoselov, K. S. The rise of graphene. Nature Mater. 6, 183-191 (2007).

7. Son, Y.-W., Cohen, M. L. \& Louie, S. G. Energy gaps in graphene nanoribbons. Phys. Rev. Lett. 97, 216803 (2006).

8. Son, Y.-W., Cohen, M. L. \& Louie, S. G. Half-metallic graphene nanoribbons. Nature 444, 347-349 (2006)

9. Han, M. Y., Özyilmaz, B., Zhang, Y. \& Kim, P. Energy band-gap engineering of graphene nanoribbons. Phys. Rev. Lett. 98, 206805 (2007).

10. Chen, Z., Lin, Y.-M., Rooks, M. J. \& Avouris, P. Graphene nano-ribbon electronics. Preprint at $<$ http://arxiv.org/abs/cond-mat/0701599> (2007)

11. Esaki, L. \& Tsu, R. Superlattice and negative differential conductivity in semiconductors. IBM J. Res Develop. 14, 61-65 (1970).

12. Tsu, R. Superlattice to Nanoelectronics (Elsevier, Oxford, UK, 2005).

13. Cottam, M. G. \& Tilley, D. R. Introduction to Surface and Superlattice Excitations (Cambridge Univ. Press, Cambridge, UK, 1989).

14. Eigler, D. M. \& Schweizer, E. K. Positioning single atoms with a scanning tunnelling microscope. Nature 344, 524-526 (1990)

15. Crommie, M. F., Lutz, C. P. \& Eigler, D. M. Confinement of electrons to quantum corrals on a metal surface. Science 262, 218-220 (1993).

16. Hiura, H. Tailoring graphite layers by scanning tunnelling microscopy. Appl. Surf. Sci. 222, 374-381 (2004).

17. Williams, J. R., DiCarlo, L. \& Marcus, C. M. Quantum Hall effect in a gate-controlled p-n junction of graphene. Science 317, 638-641 (2007).

18. Huard, B. et al. Transport measurements across a tunable potential barrier in graphene. Phys. Rev. Lett. 98, 236803 (2007)

19. Özyilmaz, B. et al. Electronic transport and quantum Hall effect in bipolar graphene p-n-p junctions. Phys. Rev. Lett. 99, 166804 (2007).

20. Marchini, S., Günther, S. \& Wintterlin, J. Scanning tunnelling microscopy of graphene on $\mathrm{Ru}(0001)$. Phys. Rev. B 76, 075429 (2007).
21. Vazquez de Parga, A. L. et al. Periodically rippled graphene: Growth and spatially resolved electronic structure. Phys. Rev. Lett. 100, 056807 (2008)

22. Pan, Y. et al. Millimeter-scale, highly ordered single crystalline graphene grown on Ru (0001) surface. Preprint at $<$ http://arxiv.org/abs/0709.2858 > (2007).

23. Matsumoto, T. \& Saito, S. Geometric and electronic structure of new carbon-network materials: Nanotube array on graphite sheet. J. Phys. Soc. Japan 71, 2765-2770 (2002).

24. Guinea, F., Katsnelson, M. I. \& Vozmediano, M. A. H. Midgap states and charge inhomogeneities in corrugated graphene. Preprint at $<$ http://arxiv.org/abs/0707.0682> (2007).

25. Ando, T. \& Nakanishi, T. Impurity scattering in carbon nanotubes—absence of back scattering. J. Phys. Soc. Japan 67, 1704-1713 (1998).

26. Ando, T., Nakanishi, T. \& Saito, R. Berry's phase and absence of back scattering in carbon nanotubes J. Phys. Soc. Japan 67, 2857-2862 (1998).

27. DiVincenzo, D. P. \& Mele, E. J. Self-consistent effective-mass theory for intralayer screening in graphite intercalation compounds. Phys. Rev. B 29, 1685-1694 (1984).

28. McEuen, P. L., Bockrath, M., Cobden, D. H., Yoon, Y.-G. \& Louie, S. G. Disorder, pseudospins, and backscattering in carbon nanotubes. Phys. Rev. Lett. 83, 5098-5101 (1999).

29. Katsnelson, M. I., Novoselov, K. S. \& Geim, A. K. Chiral tunnelling and the Klein paradox in graphene. Nature Phys. 2, 620-625 (2006).

30. Bai, C. \& Zhang, X. Klein paradox and resonant tunnelling in a graphene superlattice. Phys. Rev. B 76, 075430 (2007).

Acknowledgements

C.-H.P. thanks J. D. Sau for fruitful discussions. This research was supported by the National Science Foundation (NSF) and by the Director, Office of Science, Office of Basic Energy Science, Division of Material Sciences and Engineering, US Department of Energy (DOE). Computational resources have been provided by the NSF at the National Partnership for Advanced Computational Infrastructure and by the DOE at the National Energy Research Scientific Computing Center. Y.-W.S. was supported by the Korea Science and Engineering Foundation grant funded by the Korea government (MOST) the Korea Science and Engineering Foundation grant funded by the Kore
Correspondence and requests for materials should be addressed to S.G.L. Supplementary Information accompanies this paper on www.nature.com/naturephysics.

Reprints and permission information is available online at http://npg.nature.com/reprintsandpermissions/ 